\title{
BMJ Open GlutenSpA trial: protocol for a randomised double-blind placebo- controlled trial of the impact of a gluten- free diet on quality of life in patients with axial spondyloarthritis
}

\author{
Marion Couderc (D) , Bruno Pereira, ${ }^{2}$ Thierry Schaeverbeke, ${ }^{3}$ Thierry Thomas, ${ }^{4}$ \\ Roland Chapurlat, ${ }^{5}$ Philippe Gaudin, ${ }^{6}$ Jacques Morel, ${ }^{7}$ Maxime Dougados, ${ }^{8}$ \\ Martin Soubrier ${ }^{1}$
}

To cite: Couderc M, Pereira B, Schaeverbeke T, et al. GlutenSpA trial: protocol for a randomised doubleblind placebo-controlled trial of the impact of a gluten-free diet on quality of life in patients with axial spondyloarthritis. BMJ Open 2020;10:e038715. doi:10.1136/ bmjopen-2020-038715

- Prepublication history and supplemental material for this paper is available online. To view these files, please visit the journal online (http://dx.doi org/10.1136/bmjopen-2020038715).

Received 23 March 2020 Revised 13 August 2020 Accepted 30 September 2020

A) Check for updates

(c) Author(s) (or their employer(s)) 2020. Re-use permitted under CC BY-NC. No commercial re-use. See rights and permissions. Published by BMJ.

For numbered affiliations see end of article.

Correspondence to Dr Marion Couderc; mcouderc@chuclermontferrand.fr

\section{ABSTRACT}

Introduction Subclinical intestinal inflammation and gut dysbiosis have been reported in patients with spondyloarthritis $(\mathrm{SpA})$. In common practice, rheumatologists are increasingly confronted with patients with inflammatory rheumatism who are on gluten-free diets (GFDs), despite the lack of reliable data from controlled studies. This study aims to determine the impact of a GFD on the quality of life of patients with axial SpA. Methods and analysis The GlutenSpA study is a 24week, randomised, double-blinded, placebo-controlled, multicentre trial. Patients with axial SpA $(n=200)$ will follow a 16-week GFD and be randomly assigned (1:1) to an experimental or control arm. In the experimental arm with receive at least 6 gluten-free breads per day $+200 \mathrm{~g}$ of gluten-free penne pasta per week +6 rice flavour capsules per day. The control arm will receive at least 6 gluten-containing breads per day $+200 \mathrm{~g}$ of gluten-containing penne pasta per week +6 vital glutencontaining capsules per day. The primary end-point is the variation in Assessment of SpondyloArthritis International Society — Health Index (ASAS-HI) questionnaire between week 16 and baseline. A second open-label period of 8 weeks will follow the intervention period, during which the patient will be free to decide whether they will follow the GFD. The secondary outcomes comprise several patient-reported outcomes (SpA activity (Bath Ankylosing Spondylitis Disease Activity Index)), fatigue (Functional Assessment of Chronic Illness Therapy), depression (Hospital Anxiety and Depression Scale), functional disability index (Bath Ankylosing Spondylitis Functional Index)), variations in body mass index and Homeostasis Model Assessment Index and variations in the abundance and type of bacterial species found in the gut microbiota for a subgroup of patients $(n=40)$. The data will be analysed using the intention-to-treat principle.

\section{ETHICS AND DISSEMINATION}

The regional ethics committee (CPP Nord-ouest IV) has approved the study (IDRCB 2018-A00309-46). The results of the trial will be submitted for publication in peerreviewed journals. The authors have no relationship that may have influenced the submitted work.

\section{Strengths and limitations of this study:}

- GlutenSpA is the first randomised, double-blinded, placebo-controlled trial on the effects of a glutenfree diet on quality of life in patients with axial spondyloarthritis (SpA).

- Patients with axial spondyloarthritis will be randomised to either a 16-week gluten-free arm or a placebo arm, followed by an 8-week open-label period.

- The primary endpoint is the change in Assessment of SpondyloArthritis InternationalSociety- Health Index between baseline and week 16 .

- The secondary endpoints will include patientreported outcomes (SpA activity, fatigue, depression, functional disability index) and gut microbiota.

Trial registration number NCT04274374.

\section{BACKGROUND AND RATIONALE}

Spondyloarthritis (SpA) is a chronic inflammatory rheumatism affecting the axial skeleton and especially the sacroiliac joints. Ankylosing spondylitis is the prototype disorder. In addition to axial involvement, several other impairments are common, including arthritis, dactylitis, enthesitis, uveitis and chronic inflammatory bowel disease, defining several subgroups of SpA. ${ }^{12}$ Since the 1990s, subclinical intestinal inflammation has been described in nearly $60 \%$ of patients with $\mathrm{SpA}^{3}$ and is thought to be related to the disease activity. ${ }^{45} \mathrm{~A}$ more recent histological study of 65 patients with SpA confirmed the presence of gut inflammation in $42 \%$ of them, which was closely related to young age, male sex, SpA activity assessed by Bath Ankylosing Spondylitis Disease Activity Index (BASDAI) and axial mobility assessed 
by Bath Ankylosing Spondylitis Metrology Index. ${ }^{6}$ This gut inflammation, which can occur in macroscopically normal regions of the gut, is characterised in the acute phase by infiltrate comprising neutrophil polynuclear cells, which is gradually replaced by mononuclear cells and mediated by different types of immune cells (eg, macrophages, dendritic cells, Th1, Th17, NK lymphocytes). ${ }^{7}$ Faecal calprotectin, one of the markers commonly used to quantify gut inflammation in chronic inflammatory bowel disease (CIDI), could be increased in patients with SpA without gastrointestinal signs. ${ }^{8}{ }^{9}$ However, the link with non-steroidal anti-inflammatory drug (NSAID) use remains controversial. ${ }^{10}{ }^{11}$ Increased intestinal permeability due to gut inflammation could facilitate the passage of antigens and modulate the immune response. ${ }^{12}$ It is also enhanced by NSAIDs, the cornerstone of SpA treatment, as well as other treatments and diet. ${ }^{13}$

The microbial environment, especially the gut microbiota, has up to 100000 billion bacteria. High-throughput sequencing has identified tens of millions of bacterial genes attributed to a few thousand bacterial species that would protect the mucosal barrier from invasion by pathogens, metabolising constituents of food in useful nutrients and contribute to immune system homeostasis. Over the past 10 years, the number of publications on the association between the gut microbiota and chronic pathologies has dramatically increased, first in intestinal diseases (clostridium colitis, coeliac disease, CIDI), but also in other non-intestinal pathologies, such as diabetes, herpetiform dermatitis, nephrotic syndrome, cardiovascular diseases, autism and schizophrenia. ${ }^{1415}$

In chronic inflammatory rheumatism, whether it is SpA, psoriatic arthritis or rheumatoid arthritis, there is dysbiosis (gut microbiota imbalance) ${ }^{16}$ similar to CIDIs (decreased microbial diversity, scarcity of Firmicutes with anti-inflammatory properties). Several studies in animal models have shown a link between the gut microbiota and joint inflammation, particularly in the transgenic human leucocyte antigen (HLA) B27 rat. $^{17}$ Two very small comparative studies have reported changes in the microbiota of patients with SpA. First, a study of the faecal microbiota in 25 children with a juvenile form of $\mathrm{SpA}$ found a decrease in Firmicutes and increase in Bifidobacterium compared with 13 control patients. Another study of gut biopsies in nine patients with SpA compared with nine controls reported changes in Firmicutes and Bacteroidogenes. However, these studies need to be confirmed. $^{1819}$

The main mechanisms of action of a nutrient in inflammatory diseases have been detailed in a recent general review, particularly the direct role of food and nutrients (antioxidant effect, anti-inflammatory, immunomodulator, epigenetics, toxic) and the role of food on the gut microbiota. ${ }^{20}$ Diet clearly alters the microbiota, which has been shown for fasting ${ }^{21}$ in mice and probiotics or longchain omega-3 fatty acids ${ }^{22}$ in patients with CID.

The spectrum of gluten-related disorders has broadened and now includes coeliac disease, non-coeliac gluten sensitivity and wheat allergy. Coeliac disease is characterised by chronic enteral inflammation that causes malabsorption in genetically predisposed patients (HLA DQ2-DQ8), and alterations in the gut microbiota are thought to be involved in disease pathogenesis. This dysbiosis could be reduced by a gluten-free diet (GFD). In a study on the ileal microbiota of patients with coeliac disease, decreased abundance in Bacteroidetes and Firmicutes was reported, as described in the microbiota of patients with SpA. ${ }^{23}$ Recently, a new clinical entity has emerged called non-coeliac hypersensitivity to gluten, which is characterised by a heterogeneous clinical presentation combining intestinal and extra-intestinal signs occurring after gluten ingestion. The pathogenesis could be based on a direct toxic effect of gluten and possibly the gut microbiota. ${ }^{24}$ This entity could affect $5 \%$ of the population, but its diagnosis lacks validated criteria, and the effectiveness of a GFD varies. ${ }^{25}$

In common practice, rheumatologists are increasingly confronted with patients with inflammatory rheumatism who are dieting despite the lack of reliable data from controlled studies. A survey presented in 2015 at the Annual Congress of the French Rheumatology Society reported that nearly a quarter of patients with inflammatory rheumatism (216 RA and $166 \mathrm{SpA}$ ) followed an eviction diet, $67.1 \%$ of which were on a GFD. ${ }^{26}$ To study the effect of a diet is difficult. The placebo effect or psychological factors may contribute to the response to exclusion diets because patients know that their diet has changed. Patients with a strong belief in alternative treatments report more allergies and food intolerances than other patients and have more psychological facilities to modify their diet. This is important to consider in the design of diet studies, which require the use of randomised, double-blind, placebo trials to really answer the question. To the best of our knowledge, no studies to date have shown the effectiveness of a GFD in SpA.

\section{Objectives}

The primary objective is to determine the effects of a 16-week GFD versus placebo diet on quality of life as evaluated by the Assessment of SpondyloArthritis International Society-Health Index (ASAS-HI) questionnaire in a population of patients with axial SpA. ${ }^{27}$ The secondary objectives are to determine the effects of a 16-week GFD versus placebo diet on the activity of SpA, patientreported outcomes (pain, fatigue, depression), the tolerance to and compliance with GFD and the effect on gut microbiota. We will also determine the factors associated with the response to a GFD.

\section{Trial design}

The GlutenSpA study is a 24-week, double-blinded, placebo-controlled, multicentre, randomised trial.

The trial was approved by the French authorities (Comité de protection des Personnes Nord Ouest IV, protocol number: 2018-A00309-46). 
METHODS: PARTICIPANTS, INTERVENTIONS AND OUTCOMES

\section{Study setting}

The Rheumatology departments of seven French academic hospitals (Clermont-Ferrand, Saint-Etienne, Lyon, Grenoble, Montpellier, Cochin APHP, Bordeaux) will participate in recruitment.

\section{Patient an public involvement}

There is no patient involved in the study.

\section{Eligibility criteria}

Eligible participants must meet all of the following inclusion criteria: adult patient with a diagnosis of axial SpA as defined by the ASAS criteria ${ }^{28}$ and for which the rheumatologist does not wish to change the treatment within 4 months of inclusion, stable treatment in dose and type (NSAIDs and/or disease-modifying antirheumatic drugs (DMARDs)) for at least 3 months, but no corticosteroid infiltration in the month prior to inclusion and able to follow a GFD and provide written informed consent and submit to the requirements of the study.

Patients will be excluded if they are on any diet at the time of inclusion or within 3 months prior to inclusion; have a history of coeliac disease; received antibiotic treatment within 3 months of inclusion or are taking a probiotic; are pregnant, breastfeeding or not covered by social security or are minors or adults under the protection of the law or under the protection of justice. Furthermore, screening for serum antitransglutaminase IgA or IgG will be performed at the screening visit. Patients with serum antitransglutaminase antibodies will not be included but referred to a gastroenterologist.

\section{Interventions}

All patients $(\mathrm{n}=200)$ will be on a GFD from inclusion (day 0 (D0)) to week 16 (W16). At D0, they will be randomly allocated to two intervention groups. The experimental group (gluten-free arm, $\mathrm{n}=100$ ) will be given at least six gluten-free breads per day ( $42 \mathrm{~g}$ ) in addition to the GFD, combined with $200 \mathrm{~g}$ (raw weight) of gluten-free pasta per week and six rice flour-containing capsules per day. The control group (gluten-containing arm, $\mathrm{n}=100$ ) will be given at least six gluten-containing breads per day $(50 \mathrm{~g})$ in addition to the GFD, combined with $200 \mathrm{~g}$ (raw weight) of gluten-containing pasta per week and six vital glutencontaining capsules.

The bread and penne pasta used for the study are overthe-counter commercial products chosen for their visual resemblance and a similar taste to maximise the blindness of the study. They will be repackaged in neutral packaging. In order to reach the daily amount of gluten in a standard diet (estimated in France between 10 and $15 \mathrm{~g}$ /day) in the control arm, each day patients will have to ingest six capsules of vital gluten wheat made for the study. Vital gluten flour is an over-the-counter food supplement used to enrich a protein diet or as a base for making products, such as seitan. Capsules will be made using commercially available vital gluten and contain $0.35 \mathrm{~g}$ of gluten per

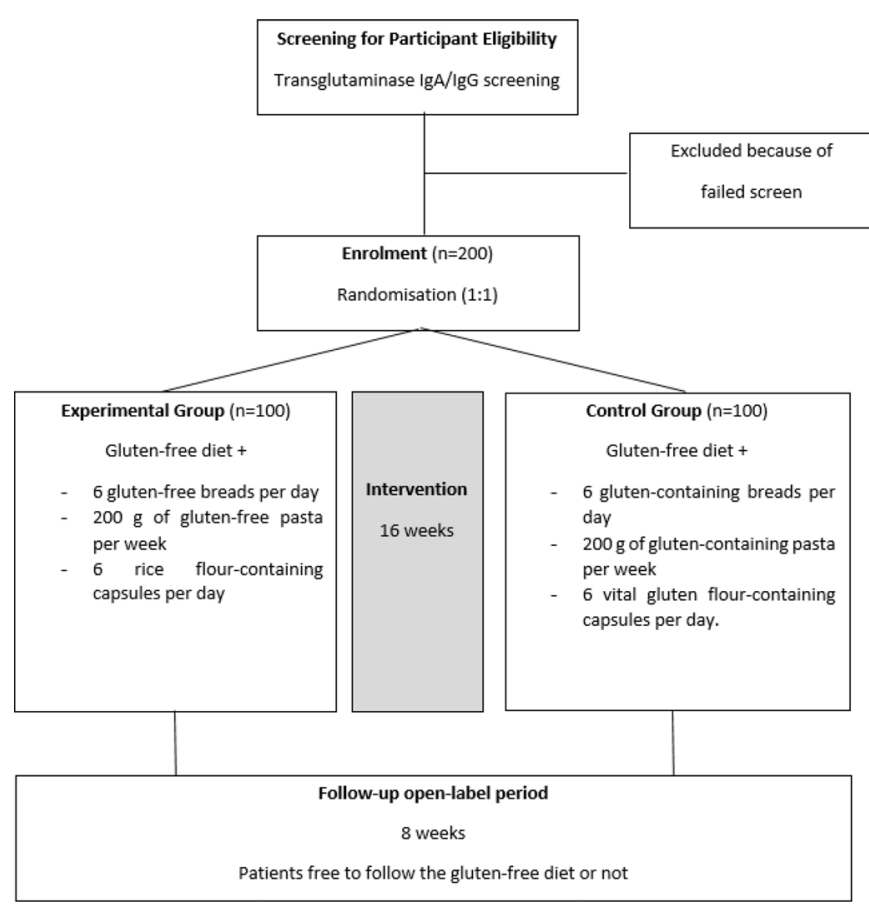

Figure 1 GlutenSpa study diagram.

capsule. The total amount of gluten in the control arm will be approximately $10.5 \mathrm{~g} /$ day. In the experimental group, patients will receive capsules containing rice flour. Capsules containing gluten and capsules containing rice flour are the same colour and size. They will be made by the central pharmacy of the University Hospital of Clermont-Ferrand from rice or gluten vital flour and sent to each centre before delivery. Breads, pasta and capsules will be furnished to each patient by the local investigating centre in two stages ( $\mathrm{S} 0$ and $\mathrm{S} 2$ ) for an 8-week period each time.

After the 16-week GFD period, patients will be offered to follow or not follow the GFD according to their own decision for an 8-week open-labelled follow-up period. The study duration for each patient is 24 weeks. The patient recruitment is expected to last 2 years (figure 1).

\section{Strategies to improve adherence}

At baseline, all participants will be told how to properly follow a GFD during a face-to-face interview. They will be given information on gluten-containing food products to avoid. The compliance with the GFD will be evaluated by the dietician at W2, W16 and W24 using a 3-day alimentary questionnaire. The alimentary questionnaire completed by the participants details all food intake during 3 days ( 2 week-days and 1 weekend day). Conversion in macronutrients (protein, fat and carbohydrate) will be completed by the dietician using Nutrilog software. Patients will be closely monitored for their nutritional balance, weight and body mass index at W2, W16 and W24.

\section{Outcome measures}

The primary outcome corresponds to the variation in the quality of life evaluated before and after the intervention 
(W16) as assessed by the ASAS-HI questionnaire. This self-reported questionnaire measures functioning and health across 17 aspects of health and 9 environmental factors, addressing categories of pain, emotional function, sleep, sexual function, mobility, self-care, community life, support/relationships, attitudes and health services. ASAS-HI has been validated in patients with radiographic and non-radiographic axial SpA. ${ }^{27}$

The secondary outcomes will be assessed before and after the intervention (W16) and the open-label 8-week period (W24):

- SpA activity evaluated by the BASDAI (D0, W16, W24).

- Functional status assessed using the Bath Ankylosing Spondylitis Functional Index (BASFI) (D0, W16, W24).

- Parameters of biological inflammation (erythrocyte sedimentation rate and C reactive protein) (D0, W16).

- Fatigue assessed by the Functional Assessment of Chronic Illness Therapy (FACIT) scale (D0, W16, W24). The FACIT is a short, 13-item, easy to administer tool that measures an individual's level of fatigue during their usual daily activities over the past week.

- Depression and anxiety assessed by the Hospital Anxiety and Depression Scale (D0, W16, W24).

- Fibromyalgia symptoms assessed using the Fibromyalgia Rapid Screening Tool (FiRST) if present at day 0 (D0, W16, W24).
- Compliance with the GFD as evaluated by an interview with the dietician to assess the follow-up of the diet (W2, W16, W24).

- Digestive discomfort assessed by a weekly digestive discomfort questionnaire (D0 to W24).

- Change in weight and body mass index (D0, W16, W24).

- Homeostasis Model Assessment (HOMA) variation: fasting blood glucose $(\mathrm{mmol} / \mathrm{L}) \times$ fasting insulin $(\mu \mathrm{mol} / \mathrm{L}) / 22.5(\mathrm{D} 0, \mathrm{~W} 16)$.

- Variations in the abundance and type of different bacterial species found in the intestinal microbiota for a subgroup of patients ( $\mathrm{n}=20$ at Clermont-Ferrand and $\mathrm{n}=20$ at Bordeaux) (D0, W16).

\section{Study visit/participant timeline}

Information about the selection, recruitment and evaluations carried out in each period is given in table 1 .

All concomitant or intercurrent medications, including SpA treatment (NSAIDs or DMARDs), will be recorded at each visit. Antibiotic use will specifically be recorded.

\section{Gut microbiota analysis}

The microbiota will be analysed in a subgroup of patients $(n=40$, the first 20 from Clermont-Ferrand and the first 20 from Bordeaux) using stool samples collected at D0 and W16, frozen at $-80^{\circ} \mathrm{C}$ and then centralised according to the following procedure:

- Extraction of the DNA contained in the faeces.

Table 1 Data collected at study visit

\begin{tabular}{|c|c|c|c|c|c|}
\hline Study period & Screening & $\begin{array}{l}\text { Enrolment } \\
\text { baseline }\end{array}$ & & Intervention & Follow-up \\
\hline Weeks & -2 & 0 & 2 & 16 & 24 \\
\hline Serum antitransglutaminase & $x$ & & & & \\
\hline Eligibility criteria & & $x$ & & & \\
\hline
\end{tabular}

Assessments

\begin{tabular}{|c|c|c|c|c|}
\hline Sociodemographic characteristics & $\mathrm{X}$ & & & \\
\hline Medical history & $\mathrm{X}$ & & & \\
\hline Concomitant medications ${ }^{\star}$ & $x$ & & $x$ & $x$ \\
\hline X-ray of the pelvis (if not available) & $X$ & & & \\
\hline Primary outcome: Quality of life (ASAS-HI) & $\mathrm{X}$ & & $\mathrm{X}$ & $\mathrm{x}$ \\
\hline Dietician consultation & $\mathrm{X}$ & $x$ & $\mathrm{X}$ & $\mathrm{x}$ \\
\hline Laboratory samples & $\mathrm{X}$ & & $x$ & \\
\hline Faecal samples: gut microbiota analysis & $\mathrm{X}$ & & $\mathrm{X}$ & \\
\hline
\end{tabular}

*Including disease modifying antirheumatic drugs, non-steroidal anti-inflammatory drug, painkillers, corticoids.

†Including Bath Ankylosing Spondylitis Disease Activity Index, Bath Ankylosing Spondylitis Functional Index, Hospital Anxiety and Depression Scale, Fibromyalgia Rapid Screening Tool, Functional Assessment of Chronic Illness Therapy and a digestive discomfort questionnaire.

ASAS-HI, Assessment of SpondyloArthritis International Society-Health Index. 
- 16S amplification (specific sequence for bacteria, highly conserved, allowing taxonomic classification).

- Sequencing of amplicons.

- Bioinformatics analysis for taxonomic assignment of the obtained sequences.

\section{Sample size}

The sample size estimation is based on a comparison between randomised groups for the change in ASAS-HI questionnaire score. According to the literature, the smallest detectable and clinically relevant change is 3.0 (22). To take into account a possible Hawthorne effect, a 2-point difference between randomisation groups is expected. Considering a two-sided type I error of $5 \%$ and statistical power of $90 \%$, an effect size of 0.5 (2-point difference for an SD between 3.5 and 4.5) can be highlighted for the change in ASAS-HI score with 87 patients per group. We propose including 200 patients (100 per group) to consider loss to follow-up.

\section{Recruitment}

Patients will be recruited from one of the seven centres participating in the study: Clermont-Ferrand, SaintEtienne, Lyon, Grenoble, Montpellier, Cochin APHP and Bordeaux. Eligible patients will be extended an offer to participate during the routine rheumatological consultation. Written informed consent will be obtained for each patient (see online supplemental file).

Each of the seven rheumatology departments is wellrecognised at the national and international levels for their expertise in managing patients with SpA. We estimate that each department is taking care of approximately 50 patients with SpA per month, so the inclusion of 1 to 2 patients per month seems to be feasible.

\section{Assignment of interventions}

Allocation

The randomisation (balanced within random block sizes) will be conducted by an investigator who is not involved in the recruitment, evaluation and/or treatment of participants.

\section{Blinding}

After assignment, study participants and care providers (rheumatologist, nurses, dieticians) will be blinded to the intervention. The analysis will also be performed under blinding. Blinding will be unlocked in case of important adverse events.

\section{Data collection, management and analysis Statistical methods}

All analyses will be performed by the Biostatistics Unit at the University Hospital of Clermont-Ferrand, which also provides methodological support for the study.

The statistical analysis will be carried out using the software programmes Stata (V.13, StataCorp, College Station, Texas, USA) and R (http:/ / cran.r-project.org/). All statistical tests will be carried out at the risk of error of the first species $\alpha$ of $5 \%$.
The primary analysis will be assessed as intention-totreat. In order to prevent attrition bias, imputation of the missing data is planned. The statistical analysis plan also provides for an additional per-protocol analysis. Continuous variables will be presented as mean and SD, subject to the normality of their distribution (Shapiro-Wilk test if necessary). In the case of skewed distribution, they will be presented as median, IQR and extreme values. Qualitative variables will be expressed as numbers and percentages. Graphic representations will be associated with these analyses as much as possible. Comparisons between groups will be systematic, ${ }^{1}$ without adjustment ${ }^{2}$ or with adjustment for factors whose distribution could be unbalanced between groups despite randomisation.

Patients will be described and compared between groups at baseline in regards to the following variables: demographic characteristics, centre, seasonality, clinical characteristics and medication. The initial comparability of the two arms will be assessed on the main characteristics of the participants and potential factors associated with the primary outcome. A possible difference between the two groups in any of these characteristics will be determined by both clinical and statistical considerations.

Deviations from the protocol and causes of abandonment will also be described. The number of patients included and the inclusion curve will be presented by group.

\section{Primary end-point analysis}

The primary endpoint will be compared between groups by the Student's t-test, or the non-parametric MannWhitney test if Student's test conditions are not met (normality verified by Shapiro-Wilk test and equality of variances by Fisher-Snedecor test). The results will be expressed in terms of effect size and 95\% CI.

\section{Secondary analysis}

In a second step, the analysis described before will be completed by a multivariate analysis of a mixed linear model type (to explain variation in the ASAS-HI score) in order to take into account the covariates retained in the univariate analysis for their clinical relevance (stratification criterion: sex, age, duration of disease, smoking, type of SpA medication (eg, NSAID or TNF blockers), BASDAI and BASFI scores at baseline, FiRST score at baseline) or the centre effect (considered random). The results will be expressed in terms of regression coefficients and 95\% CIs. The normality of the residues will be studied; if necessary, a logarithmic transformation of the dependent variable will be proposed.

Comparisons between the groups will be performed ${ }^{1}$ in a similar manner as previously presented for quantitative secondary endpoints and ${ }^{2}$ the chi-squared or Fisher exact test for categorical variables. Concerning the study of factors associated with a good response to the regimen, defined by a variation of the ASAS-HI score of at least 2 points, comparisons in univariate situations will resume the statistical analyses described previously. 
We will study more precisely the sex, the characteristics of the SpA (HLA-B27, inflammatory anomaly with MRI of the sacroiliac, duration of the disease), the use of antibiotics during the study, body mass index and variations in weight, the type of initial abnormality in the intestinal flora and immunological profile. The multivariate analysis will consider a logistic regression for which covariates will be determined based on univariate results of clinical relevance. The results will be expressed in terms of ORs and $95 \%$ CIs.

\section{DISCUSSION}

This is the first randomised, double-blind, placebocontrolled clinical trial on the effect of a GFD on quality of life in SpA. Given the popularity of GFDs in patients with inflammatory rheumatisms, data on the efficacy and safety are needed.

The strength of this study lies in its placebo-controlled design, which appears necessary given the importance of the placebo effect of diets. Such an ambitious study could give highlights for understanding the links between symptoms, disease, diet and microbiome. If this study demonstrates a beneficial effect of the GFD in patients with axial $\mathrm{SpA}$, it could lead to recommendations in current practice or new therapeutics targeting such a manipulation of the microbiome. If it is negative, it will provide an answer to the frequent questions of patients on the benefit of a GFD in this disease.

Regarding the study of the microbiota, it will provide new data on the microbiota of patients with SpA and aid in understanding the interaction between a GFD and the microbiota.

Like other studies on diets, the major limitation of the study will be the possible difficulties in adherence to the GFD. To minimise this potential bias, the dieticians will collect the amount of bread, pasta and pills ingested during each period and three face-to-face dietetic consultations (W0, W2 and W16) are planned to educate the patients.

\section{TRIAL STATUS}

At the time of initial manuscript submission, recruitment had not started and is expected to begin in September 2020. The last patient is expected to be included in March 2022.

\footnotetext{
Author affiliations

${ }^{1}$ Rheumatology, CHU Clermont-Ferrand, Clermont-Ferrand, France

${ }^{2}$ Biostatistical Unit, CHU Clermont-Ferrand, Clermont-Ferrand, France

${ }^{3}$ Rheumatology, CHU de Bordeaux, Bordeaux, Aquitaine, France

${ }^{4}$ Rheumatology, CHU ST ETIENNE, Saint Etienne, France

${ }^{5}$ Rheumatology, CHU Lyon, Lyon, Auvergne-Rhône-Alpes, France

${ }^{6}$ Rheumatology, CHU Grenoble Alpes, Grenoble, Rhône-Alpes, France

${ }^{7}$ Rheumatology, CHU Montpellier, Montpellier, Languedoc-Roussillon, France

${ }^{8}$ Rheumatology, Cochin Institute, Paris, Île-de-France, France
}

Twitter Maxime Dougados @rhuematoid arthritis
Contributors CM, PB and SM designed and revised critically for important intellectual content of the project; approved the final version of the work and agreed to be accountable for all aspects of the work in ensuring that questions related to the accuracy or integrity of any part of the work are appropriately investigated and resolved. ST, TT, CR, GP, MJ and DM revised critically for important intellectual content of the project; approved the final version of the work and agreed to be accountable for all aspects of the work in ensuring that questions related to the accuracy or integrity of any part of the work are appropriately investigated and resolved.

Funding This study was supported by the 'Direction Générale de l'Offre de Soins' (DGOS)(regional multicentric PHRC COUDERC 2017).

Competing interests None declared.

Patient consent for publication Not required.

Provenance and peer review Not commissioned; externally peer reviewed.

Supplemental material This content has been supplied by the author(s). It has not been vetted by BMJ Publishing Group Limited (BMJ) and may not have been peer-reviewed. Any opinions or recommendations discussed are solely those of the author(s) and are not endorsed by BMJ. BMJ disclaims all liability and responsibility arising from any reliance placed on the content. Where the content includes any translated material, BMJ does not warrant the accuracy and reliability of the translations (including but not limited to local regulations, clinical guidelines, terminology, drug names and drug dosages), and is not responsible for any error and/or omissions arising from translation and adaptation or otherwise.

Open access This is an open access article distributed in accordance with the Creative Commons Attribution Non Commercial (CC BY-NC 4.0) license, which permits others to distribute, remix, adapt, build upon this work non-commercially, and license their derivative works on different terms, provided the original work is properly cited, appropriate credit is given, any changes made indicated, and the use is non-commercial. See: http://creativecommons.org/licenses/by-nc/4.0/.

\section{ORCID iD}

Marion Couderc http://orcid.org/0000-0002-2001-1132

\section{REFERENCES}

1 López-Medina C, Moltó A. Update on the epidemiology, risk factors, and disease outcomes of axial spondyloarthritis. Best Pract Res Clin Rheumatol 2018;32:241-53.

2 Sieper J, Poddubnyy D. Axial spondyloarthritis. Lancet 2017;390:73-84.

3 Mielants H, Veys EM, Cuvelier C, et al. The evolution of spondyloarthropathies in relation to gut histology. II. histological aspects. J Rheumatol 1995;22:2273-8.

4 Mielants H, De Vos M, Cuvelier C, et al. The role of gut inflammation in the pathogenesis of spondyloarthropathies. Acta Clin Belg 1996;51:340-9.

5 De Vos M, Mielants H, Cuvelier C, et al. Long-Term evolution of gut inflammation in patients with spondyloarthropathy. Gastroenterology 1996;110:1696-703.

6 Van Praet L, Van den Bosch FE, Jacques P, et al. Microscopic gut inflammation in axial spondyloarthritis: a multiparametric predictive model. Ann Rheum Dis 2013;72:414-7.

7 Jacques $P$, Elewaut $D$. Joint expedition: linking gut inflammation to arthritis. Mucosal Immunol 2008;1:364-71.

8 Klingberg E, Carlsten H, Hilme E, et al. Calprotectin in ankylosing spondylitis--frequently elevated in feces, but normal in serum. Scand $J$ Gastroenterol 2012;47:435-44.

9 Matzkies FG, Targan SR, Berel D, et al. Markers of intestinal inflammation in patients with ankylosing spondylitis: a pilot study. Arthritis Res Ther 2012;14:R261.

10 Klingberg $\mathrm{E}$, Strid $\mathrm{H}$, Ståhl $\mathrm{A}$, et al. A longitudinal study of fecal calprotectin and the development of inflammatory bowel disease in ankylosing spondylitis. Arthritis Res Ther 2017:19:21.

11 Moreno Martínez MJ, Moreno Ramos MJ, Linares Ferrando LF. Relationship between fecal calprotectin, anti-Saccharomyces cerevisiae antibodies and other markers of disease activity in patients with spondyloarthritis. Reumatol Clin 2019;15:360-2.

12 Dignass AU. Mechanisms and modulation of intestinal epithelia repair. Inflamm Bowel Dis 2001;7:68-77.

13 Lopetuso LR, Scaldaferri F, Bruno G, et al. The therapeutic management of gut barrier leaking: the emerging role for mucosal barrier protectors. Eur Rev Med Pharmacol Sci 2015;19:1068-76. 
14 Strati F, Cavalieri D, Albanese D, et al. New evidences on the altered gut microbiota in autism spectrum disorders. Microbiome 2017;5:24.

15 El-Chammas K, Danner E. Gluten-Free diet in nonceliac disease. Nutr Clin Pract 2011;26:294-9.

16 Breban M. Gut microbiota and inflammatory joint diseases. Joint Bone Spine 2016;83:645-9.

17 Taurog JD, Maika SD, Satumtira N, et al. Inflammatory disease in HLA-B27 transgenic rats. Immunol Rev 1999;169:209-23.

18 Costello M-E, Ciccia F, Willner D, et al. Brief report: intestinal dysbiosis in ankylosing spondylitis. Arthritis Rheumatol 2015;67:686-91.

19 Gevers D, Kugathasan S, Denson LA, et al. The treatment-naive microbiome in new-onset Crohn's disease. Cell Host Microbe 2014;15:382-92.

20 Varoquier $\mathrm{C}$, Salmon J-H, Danion F, et al. Les effets des régimes et des stratégies diététiques dans La polyarthrite rhumatoïde. Rev Rhum 2015;82:283-90.

21 Carmody RN, Gerber GK, Luevano JM, et al. Diet dominates host genotype in shaping the murine gut microbiota. Cell Host Microbe 2015;17:72-84

22 Uranga JA, López-Miranda V, Lombó F, et al. Food, nutrients and nutraceuticals affecting the course of inflammatory bowel disease. Pharmacol Rep 2016;68:816-26.

23 Wacklin P, Laurikka P, Lindfors K, et al. Altered duodenal microbiota composition in celiac disease patients suffering from persistent symptoms on a long-term gluten-free diet. Am J Gastroenterol 2014:109:1933-41.

24 Makharia A, Catassi C, Makharia GK. The overlap between irritable bowel syndrome and non-celiac gluten sensitivity: a clinical dilemma. Nutrients 2015:7:10417-26.

25 Isasi C, Stadnitsky A, Casco F, et al. Non-Celiac gluten sensitivity and chronic refractory low back pain with spondyloarthritis features. Med Hypotheses 2020;140:109646.

26 Albert C, Brocq O, Berthier R, et al. Enquête sur l'utilisation d'un régime d'éviction auprés des patients atteints d'un rhumatisme inflammatoire chronique : propos de 382 patients. Paris: $28 \mathrm{eme}$ congrès de Rhumatologie, 2015.

27 Kiltz U, van der Heijde D, Boonen A, et al. Measurement properties of the ASAS health index: results of a global study in patients with axial and peripheral spondyloarthritis. Ann Rheum Dis 2018;77:1311-7.

28 Rudwaleit M, van der Heijde D, Landewé R, et al. The assessment of spondyloarthritis International Society classification criteria for peripheral spondyloarthritis and for spondyloarthritis in general. Ann Rheum Dis 2011;70:25-31.

29 Kiltz U, van der Heijde D, Boonen A, et al. Measuring impairments of functioning and health in patients with axial spondyloarthritis by using the ASAS health index and the environmental item set: translation and cross-cultural adaptation into 15 languages. $R M D$ Open 2016;2:e000311 